\section{Neutron Terminology}

Weinberg and Wigner ${ }^{1}$ have made a plea for a more scholarly exposition of reactor technology; Ghose and Young ${ }^{2}$ suggest that the proposed introduction of a unit of neutron flux be made an opportunity to make customary the use of the word 'flux' in this context. Their suggestion, however, involves an over-simplification, through neglecting to make allowance for the two concepts of neutron current and neutron flux, the importance of which $I$ have emphasized elsewhere ${ }^{3}$.

In reactor terminology, neutrons traversing unit area in unit time are measured as neutron current, not as neutron flux. It would be more correct to say neutron current density, but there is no good reason to prefer flux density. Neutron flux has, conventionally, the same dimensions as neutron current, but arises from a different point of view : whereas neutron current is a rate of crossing a surface by neutrons, neutron flux is a rate of track formation by the neutrons in a volume, being equal to the neutron density (in the sense of concentration) times the mean speed. If Ghose and Young's suggestion of calling this quantity 'flux density' were adopted, then flux would become total number of neutrons times mean speed-a change that would make confusion worse confounded. It should be noted that though a unit of flux would, on dimensional considerations alone, be valid for neutron current also, this extension of its use would lead to a possibility of error because, in going from flux to current, a factor of $\frac{1}{2}$ appears (for random motion) as a result of the inclination of the line of flight to a surface becoming involved.

While feeling that the meaning at present attached to 'neutron flux' can be justified, there is another usage which cannot. Whereas a microscopic crosssection has dimensions $L^{2}$, the so-called macroscopic cross-section has dimensions $L^{-1}$ : it is not a crosssection at all. It can, for example, be an absorption coefficient, and has the correct dimensions for one. Terms such as 'absorption coefficient' are already well known, are less cumbersome, and using them instead of macroscopic cross-section would permit the word 'microscopic' to be dropped when referring to a true cross-section.

It would, furthermore, lead to greater clarity if 'multiplication constant' were reserved for a regular lattice of fuel and moderator of infinite extent, and not used for the most important variable in a real reactor. For the latter, 'reproduction ratio' seems the appropriate choice.

\section{Burton Court,} London, S.W.3.

' Weinberg, A. M.. and Wigner, F. P., The Physical Theory of Neutron Chain Reactors (Univ. Chicago Press, 1958).

8 Ghose, S. C., and Young, J. C., Nature, 190, 621 (1961).

- Harper, W. R., Basic Principles of Fission Reactors (Interscience Pub., New York and London, 1961).

\section{Neutron 'Flux'}

IT is the title, not the definition, of 'neutron flux' which is ambiguous, particularly the application of a vector term to a scalar quantity. The title should imply some connexion with the flow of neutrons; in fact, as the velocities are integrated regardless of direction, the function defines the rate of generation of neutron track-lengths per unit volume at a given point. This is important in reaction problems because, as the elementary particles are treated as spherical and hence presenting a fixed cross-section to any neutron, it measures the collision potential. It is nothing whatever to do with the flow of neutrons, except in such special cases as collimated beams or by indirect calculation when the distribution pattern has been determined; yet the phrase gives this impression and misleads many entrants to the nuclear field.

The mere fact that neutron flux, like the pressure or temperature of a gas, is caused by the movement of the particles involved, is no good reason for applying to a scalar a title which in all other branches of science is reserved for vectors; my own preferred alternative is noutron (collision) potential, but even a synthetic proposal would be better than the present term.

K. L. Stretch

\section{College of Advanced Technology,}

Gosta Green, Birmingham 4.

\section{CRYSTALLOGRAPHY}

\section{'Effective' Ionic Charge in Crystals}

A ReCent communication by Mooser and Pearson ${ }^{1}$ has directed attention to the confusion which ean result from two distinct usages of the term 'ionic'. As an example they cite discussions of the bonding in zinc blende, where the tetrahedral bonds may be said to arise from resonance involving (i) $\mathrm{Zn}^{2-} \mathrm{S}^{2+}$ and (ii) $\mathrm{Zn}^{2+} \mathrm{S}^{2-}$. The first is the extreme covalent structure which gives each atom four valence electrons; the second is conventionally called the fully ionic structure since both atoms have closed-shell configurations. As Mooser and Pearson point out, both (i) and (ii) are physically ionic, and the convention which describes 'neutral bonded' $\mathrm{Zn}^{\circ} \mathrm{S}^{0}$ as 50 per cent ionic, because it involves equal resonance contributions from (i) and (ii), can be most misleading.

The question of the ionic charge in compounds isomorphous with zine blende, a class which includes several important semi-conductors, has been much discussed, since for such purposes as energyband calculations or the calculation of electronic levels at a vacancy it is necessary to know the electrostatic potential in the crystal. There is considerable confusion in the literature on the question of how this ionic charge may be measured. The confusion is due partly to the situation to which Mooser and Pearson have directed attention, but is also a result of the use of the term 'effective ionic charge'. Since these authors also use this expression without defining it, their timely article will not decrease the confusion on this point. As an example of the way in which effective ionic charge can be determined physically, they instance the dipole moment $\mu$ of a hydrogen halogenide molecule for which the nuclear separation is $r_{0}$. The effective ionic charge is then taken to be $Z e=\mu / r_{0}$, from which it is apparent that the definition of effective ionie charge which thay intend may be "the value for the ionic charge deduced from a physical measurement on the basis of an over-simplified model or theory". The purpose of the present communication is to point out that the effective charge which can be deduced from certain physical measurements on crystals is clearly not the ionic charge, although it has been used as such ${ }^{2}$.

I shall confine attention to crystals isomorphous with zinc blende. Methods of measuring the ionic charge which have been suggested may be classed as : (a) miscellaneous, (b) X-ray diffraction, and $(c)$ 